\title{
Hepatocellular Carcinoma Screening With Computed Tomography Using the Arterial Enhancement Fraction With Radiologic-Pathologic Correlation
}

\author{
Adrian Thomas Huber, MD, *† Frederik Schuster, MD, * Lukas Ebner, MD, * + Yanik Bütikofer, MD, * \\ Daniel Ott, MD, * Lars Leidolt, MD, * Andreas Jöres, MD, * Matteo Montani, MD, $\S$ \\ Johannes Heverhagen, MD, * and Andreas Christe, MD*
}

Objective: The aim of this study was to investigate the performance of the arterial enhancement fraction (AEF) in multiphasic computed tomography (CT) acquisitions to detect hepatocellular carcinoma (HCC) in liver transplant recipients in correlation with the pathologic analysis of the corresponding liver explants. Materials and Methods: Fifty-five transplant recipients were analyzed: 35 patients with 108 histologically proven $\mathrm{HCC}$ lesions and 20 patients with end-stage liver disease without HCC. Six radiologists looked at the triphasic CT acquisitions with the AEF maps in a first readout. For the second readout without the AEF maps, 3 radiologists analyzed triphasic $\mathrm{CT}$ acquisitions (group 1), whereas the other 3 readers had 4 contrast acquisitions available (group 2). A jackknife free-response reader receiver operating characteristic analysis was used to compare the readout performance of the readers. Receiver operating characteristic analysis was used to determine the optimal cutoff value of the AEF.

Results: The figure of merit $(\theta=0.6935)$ for the conventional triphasic readout was significantly inferior compared with the triphasic readout with additional use of the AEF $(\theta=0.7478, P<0.0001)$ in group 1 . There was no significant difference between the fourphasic conventional readout $(\theta=0.7569)$ and the triphasic readout $(\theta=0.7615, P=0.7541)$ with the $\mathrm{AEF}$ in group 2 . Without the AEF, HCC lesions were detected with a sensitivity of $30.7 \%$ (95\% confidence interval $[\mathrm{CI}], 25.5 \%-36.4 \%)$ and a specificity of $97.1 \%(96.0 \%-98.0 \%)$ by group 1 looking at $3 \mathrm{CT}$ acquisition phases and with a sensitivity of $42.1 \%$ $(36.2 \%-48.1 \%)$ and a specificity of $97.5 \%(96.4 \%-98.3 \%)$ in group 2 looking at $4 \mathrm{CT}$ acquisition phases. Using the AEF maps, both groups looking at the same 3 acquisition phases, the sensitivity was $47.7 \%$ (95\% CI, $41.9 \%-53.5 \%)$ with a specificity of $97.4 \%(96.4 \%-98.3 \%)$ in group 1 and $49.8 \%(95 \%$ CI, $43.9 \%-55.8 \%) / 97.6 \%(96.6 \%-98.4 \%)$ in group 2 . The optimal cutoff for the AEF was $50 \%$.

Conclusion: The AEF is a helpful tool to screen for HCC with CT. The use of the AEF maps may significantly improve HCC detection, which allows omitting the fourth $\mathrm{CT}$ acquisition phase and thus making a $25 \%$ reduction of radiation dose possible.

Key Words: computed tomography, liver CT perfusion,

hepatic nodule detection rate, HCC screening, radiation dose reduction,

orthotopic liver transplantation

(Invest Radiol 2016;51: 25-32)

$\mathrm{H}$ epatocellular carcinoma (HCC) is the fifth most frequent cancer and the second cause of cancer deaths worldwide. ${ }^{1}$ If an HCC is

Received for publication May 18, 2015; and accepted for publication, after revision, July 3,2015 .

From the *Department of Diagnostic, Interventional and Pediatric Radiology, University Hospital Inselspital Bern, Bern, Switzerland; $\uparrow$ Department of Polyvalent and Oncologic Radiology, University Hospital Pitié-Salpêtrière, Paris, France; †Department of Radiology, Duke University Medical Center, Durham, North Carolina; and §Institute of Pathology, University of Bern, Bern, Switzerland.

Conflicts of interest and sources of funding: none declared.

Reprints: Adrian Thomas Huber, MD, Department of Diagnostic, Interventional, and Pediatric Radiology, University Hospital Inselspital Bern, CH-3010 Bern, Switzerland. E-mail: adrian.huber@insel.ch.

Copyright (C) 2015 Wolters Kluwer Health, Inc. All rights reserved.

ISSN: 0020-9996/16/5101-0025

DOI: $10.1097 /$ RLI.0000000000000201 detected early, patients can be cured in up to $90 \%$ of the cases under the best circumstances. ${ }^{2}$ If not, survival rate lies between several years for patients undergoing liver transplantation and several months for patients without treatment options. ${ }^{3}$ The most cost-effective strategy to screen patients with chronic liver disease for $\mathrm{HCC}$ is sonography with testing of the serum alpha-fetoprotein level every 6 months. ${ }^{4}$ But a low sensitivity of ultrasound for HCC detection favors screening with computed tomography (CT), especially for patients on the orthotopic liver transplantation (OLT) list. ${ }^{5,6}$

During the last few years, there were several published papers showing an increased detectability of $\mathrm{HCC}$ lesions with CT perfusion. ${ }^{7-10}$ Beneath the motion artefacts, 1 major limitation for CT perfusion is the high radiation dose, especially in a setting of long-duration HCC surveillance in patients on the OLT list. ${ }^{11}$ The calculation of the arterial enhancement fraction (AEF), as introduced by Kim et al, ${ }^{12}$ allows the calculation of a quantitative colored liver map using the raw data of a standard CT scan with triphasic acquisition (unenhanced, arterial, and portal venous phase). The AEF is calculated as the proportion of the arterial to the portal venous attenuation, normalized by the underlying unenhanced liver attenuation: $\mathrm{AEF}=\left[\left(\mathrm{HU}_{\mathrm{A}}-\mathrm{HU}_{\mathrm{U}}\right) /\left(\mathrm{HU}_{\mathrm{P}}-\right.\right.$ $\left.\left.\mathrm{HU}_{\mathrm{U}}\right)\right] \times 100$, where $\mathrm{HU}$ is the attenuation, $\mathrm{A}$ is the arterial phase, $\mathrm{P}$ is the portal phase, and $\mathrm{U}$ is the unenhanced $\mathrm{CT}$ acquisition. An HCC lesion that has a predominantly arterial blood supply is expected to show a high AEF, in contrast to normal liver tissue with around $20 \%$ arterial blood and $80 \%$ portal venous blood supply. ${ }^{13}$

Kim et al showed a very high sensitivity of $88.8 \%$ for $\mathrm{HCC}$ detection using the AEF maps. But they included only HCC lesions detected on imaging studies, proven positive by surgery, biopsy, or the recommendations from the American Association for the Study of Liver Disease (AASLD) criteria. ${ }^{14}$ The rest of the liver parenchyma, potentially containing other lesions that were not visible or not detected on imaging studies, was considered normal without histologic correlation. Also, patients with prior transarterial chemoembolization (TACE) were excluded. This is a major weakness of their study because radiologists regularly see CT scans of patients with advanced disease on the OLT waiting list that have undergone previous TACE.

The aim of this study was to investigate the AEF in a realistic HCC-surveillance setting in patients with advanced liver cirrhosis on the OLT waiting list. These results should be compared with the histologic analysis of the whole liver explants serving as the gold standard. Because the AEF tool uses a triphasic image acquisition, another assumption would be if the use of the AEF tool would supersede the acquisition of 4 contrast phases (unenhanced, arterial, portal venous, and equilibrium phase) and thus allow a $25 \%$ reduction in radiation dose. Furthermore, a comparison of the screening performance of the AEF tool in liver segments with and without previous TACE was intended.

\section{MATERIALS AND METHODS}

This retrospective study received approval from the local ethics committee. Transplantation candidates were selected by the MELD and Milan criteria. ${ }^{15,16}$ All patients who underwent liver transplantation between 2010 and 2014 at a tertiary care institution were 
reviewed. Included were patients with liver cirrhosis and pathologically proven HCC as well as patients with liver cirrhosis but no HCC, which was also pathologically proven. Excluded patients were those with no cirrhosis, no available multiphase CT of the liver in the 4 months before the liver transplantation, or patients with hepatic interventions between the last liver CT and the transplantation, including surgery, endovascular, and transcutaneous interventions to the liver. The result was a total of 55 patients: 35 patients with liver cirrhosis and a total of 108 pathologically proven HCC lesions, as well as 20 patients with liver cirrhosis where pathological analysis of the explant excluded any HCC lesion. Of the 108 HCC lesions, 47 lesions were already treated by previous TACE, whereas 61 lesions remained without treatment at the time of the liver transplantation. Twenty-seven HCC lesions were larger than $2 \mathrm{~cm}$, whereas 81 lesions measured $2 \mathrm{~cm}$ or less in long axis diameter. Seventeen HCC lesions larger than $2 \mathrm{~cm}$ were treated with TACE before the CT acquisitions, whereas 10 of the lesions larger than $2 \mathrm{~cm}$ were not treated with TACE before the CT acquisition and the liver transplantation. All inclusion and exclusion criteria as well as the resulting HCC lesions are summarized in Figure 1.

\section{Mapping of HCC Lesions}

After liver transplantation, all the liver explants were systematically analyzed by a hepatopathologist. First, he analyzed the gross specimen macroscopically by cutting it into 1-cm-thick slices. Every macroscopically suspect nodule was histologically analyzed and categorized as HCC or no HCC. Well-differentiated HCCs were termed HCC lesions, whereas regenerative and dysplastic nodules were termed no HCC. In a second step, all the HCC lesions defined by histopathologic analysis were matched with the CT scans and localized on a segmented liver scheme, considered as the true-positive HCC lesions; this was done by 2 radiologists with 5 and 15 years' experience in abdominal radiology. Imaging characteristics of the lesions on the CT acquisitions were not taken into account. Nodules with some imaging characteristics of HCC but declared negative by the hepatopathologist were considered as no HCC. These were mainly nodules presenting arterial enhancement without washout, mostly consistent with regenerative or dysplastic nodules.

\section{Image Acquisition}

All the CT scans were acquired in 4 contrast phases: unenhanced before intravenous contrast administration, in the arterial phase at 35 seconds after intravenous contrast administration, in the portal venous phase at 70 seconds, and in the equilibrium phase at 3 minutes. All the CT acquisitions were performed on a Siemens Somatom Definition Flash, a Siemens Somatom Definition Edge (Siemens Healthcare, Erlangen, Germany), or a Philips CT Brilliance 64 CT scanner (Philips Medical Systems, Best, the Netherlands). Tube voltage was set at $120 \mathrm{kVp}$ with automated tube current modulation. Axial slices 1 and $5 \mathrm{~mm}$ thick were reconstructed. The AEF was calculated using the 1-mm unenhanced, arterial, and portal venous CT acquisitions, using the AEF tool contained in the CT oncology mode on a syngo.via workstation by Siemens Healthcare (Erlangen, Germany).

\section{Image Analysis}

Six radiologists with 3 to 8 years' experience in abdominal radiology, who did not have clinical information other than known liver cirrhosis, analyzed the CT images in 2 readout rounds. The 2 radiologists who matched the histopathological results with the CT images did not participate in this readout. The first readout was done with the AEF perfusion maps available and the second readout without AEF maps. The second readout was performed at least 4 weeks after the first readout. The readout round with the AEF tool was performed before the readout round without the AEF tool to ensure that an eventually occurring recognition bias did not favor the AEF tool. For the first readout, the readers looked at the triphasic grayscale images in conjunction with the color AEF maps. The fourth acquisition phase (equilibrium phase) was not available to any of the readers during the first readout round. The AEF maps were shown in axial slices alone, as well as an overlay of the AEF maps with the unenhanced grayscale CT images (30\% AEF map and $70 \%$ grayscale map) in axial, coronal, and sagittal reconstructions. The polygonal region of interest (ROI) was set as large as possible in the lesion on the AEF perfusion map, excluding eventual zones of necrosis and major vessels. The readers were instructed not to measure ROIs smaller than $5 \mathrm{~mm}$ whenever possible. A $1 \times 1 \mathrm{~cm}$ reference ROI was set in the normal parenchyma of each liver without containing

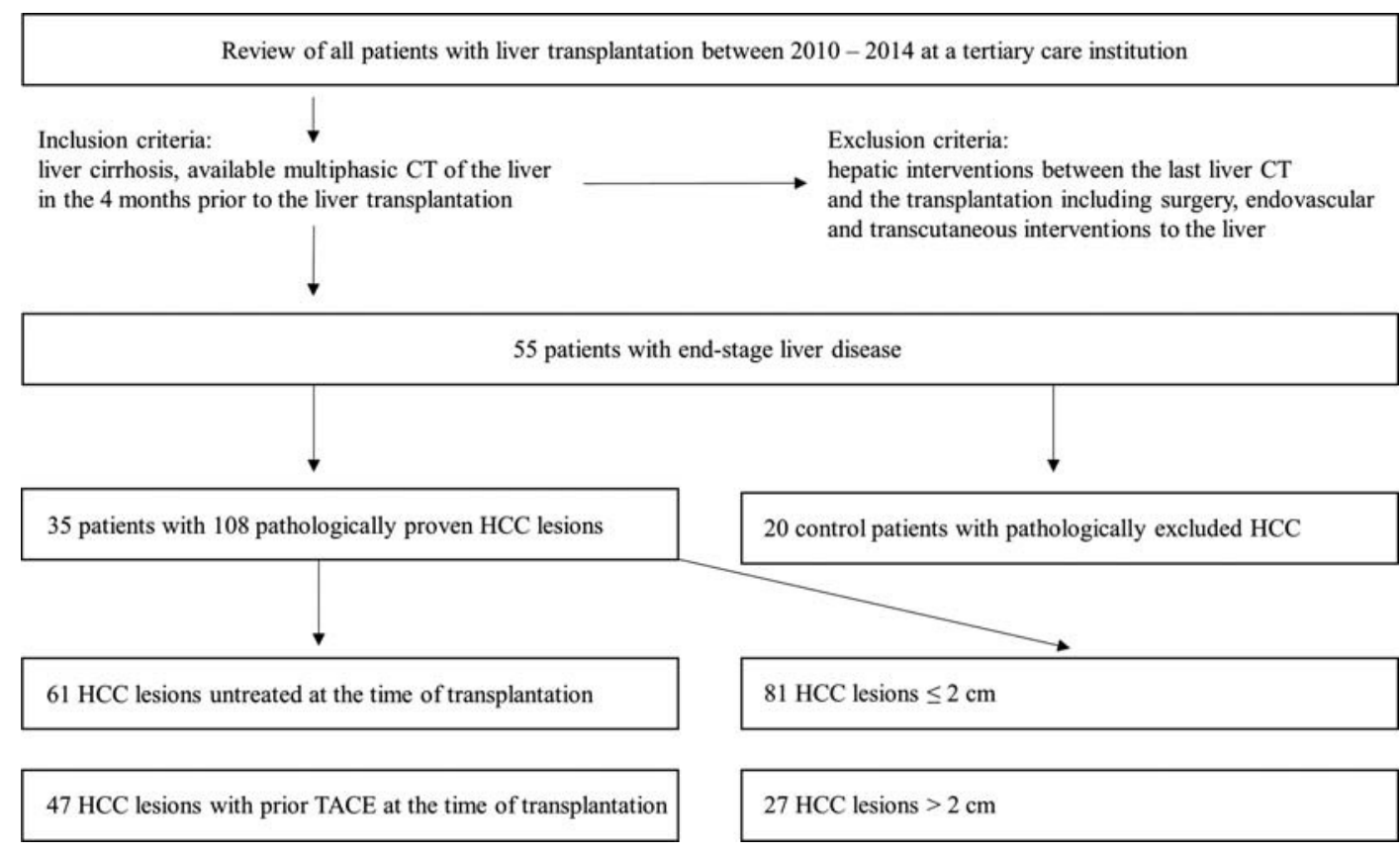

FIGURE 1. Inclusion and exclusion criteria of the patient population with the resulting HCC lesions. 
major vessels or liver lesions to record background liver tissue enhancement characteristics. The readers noted the AEF values (in percentage) on an Excel spreadsheet and allocated the lesions on the segmented liver scheme. Each lesion was assigned a confidence number between 1 and 5 (1, minimal linear or point-shaped arterial enhancement, no washout; 2, weak, laminar arterial enhancement, no washout; 3, arterial enhancement, uncertain washout or no arterial enhancement and clear washout; 4, weak arterial enhancement, clear washout; 5, strong arterial enhancement, clear washout). The AEF perfusion maps and measurements were used to either downgrade or upgrade the confidence of the conventional grayscale images. Confidence 1 to 2 was considered as "no HCC lesion" and confidence 3 to 5 was considered as "HCC lesion."

For the second readout round without the AEF perfu sion maps, the readers were separated into 2 groups with 3 readers each. To compare triphasic with fourphasic acquisitions, the first group received 3 CT acquisitions to evaluate (unenhanced, arterial, and portal venous phase), whereas the second group had $4 \mathrm{CT}$ acquisitions available (unenhanced, arterial, portal venous, and equilibrium phase).

\section{Statistics}

A receiver operating characteristic (ROC) analysis was used to calculate the optimized cutoff values for the measured AEF values using the MedCalc software, version 7.6.0.0. (Mariakerke, Belgium). ${ }^{17}$ The ROC analysis was performed on a per-segment basis using the measured AEF values on the AEF map in suspected HCC lesions. The control ROIs measured in normal hepatic tissue in each AEF map were assigned to the segments considered as normal by the readers. Each segment was considered either as true-positive or true-negative for HCC as defined by the hepatopathologist as the gold standard.

A jackknife free-response reader ROC (JAFROC) analysis using the confidence levels given by the readers for each identified lesion (1-5) was done to compare the 2 readouts. The analysis was performed with a JAFROC analysis software, version 4.2.1 (available at http://www.devchakraborty.com). ${ }^{18}$ The 2 groups of readers were analyzed separately: the triphasic readout with AEF compared with the triphasic conventional readout without AEF for group 1 and the triphasic readout with AEF compared with the fourphasic readout without AEF for group 2. To compare 3 versus 4 phases' detectability, a paired JAFROC analysis was performed. Because the true-positive lesions have to be identical for JAFROC analysis, a comparison of segments with previous TACE and segments without previous TACE was not possible with this method. Instead, sensitivity, specificity and positive predictive value (PPV) were calculated on a per-segment basis with a contingency $2 \times 2$ table analysis.

To compare the serum alpha-fetoprotein levels with the measured AEF values and the pathologic tumor mass, a Spearman rank correlation coefficient was calculated. The following variables have been compared: the serum alpha-fetoprotein level of each patient, the sum of the diameters of lesions with a mean AEF of $50 \%$ and more, in centimeters, and the sum of the HCC diameters as measured by the pathologist in each patient.

For interreader variability, a nonweighted binary $\kappa$-statistic for multiple readers was calculated ( $\kappa$ value $0-0.2$, poor; $0.21-0.4$, fair; $0.41-0.6$, moderate; $0.61-0.8$, substantial; $0.81-1$, almost perfect), ${ }^{19,20}$ using the MedCalc software. ${ }^{17}$

\section{RESULTS}

\section{Optimal Cutoff and Performance of the AEF}

Based on the present patient population, the optimal cutoff AEF value was $50 \%$ to discriminate HCC from non-HCC lesions with a sensitivity of $94.6 \%$ (95\% confidence interval [CI] 91.3\%-97.0\%)
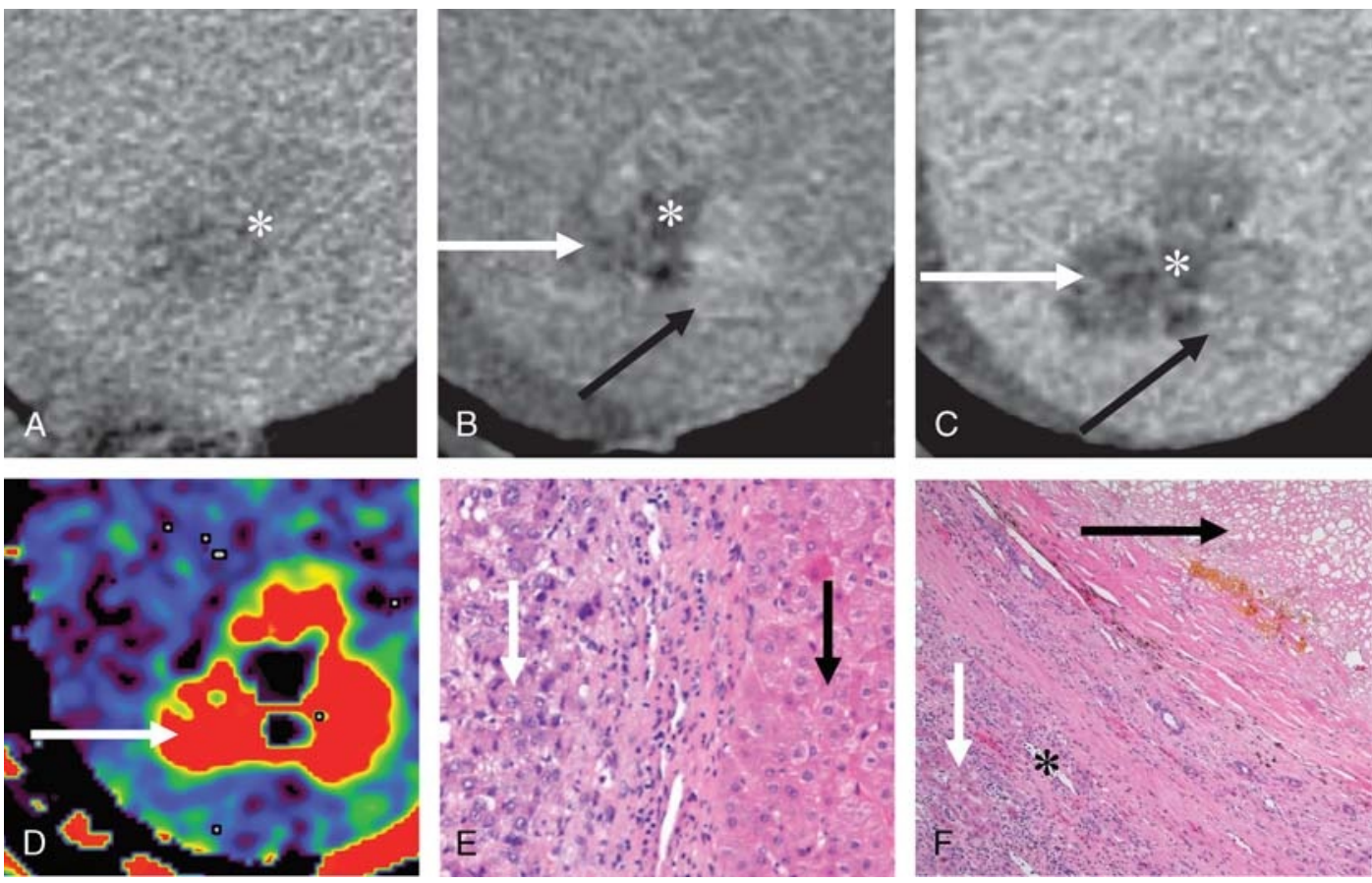

FIGURE 2. Typical HCC with central tumor necrosis (A, B, C; white asterisk), which can also be seen on the corresponding histopathologic image (F; black arrow). The anterior and medial tumor borders show nodular arterial enhancement with washout in the portal venous phase (B, $C$; black arrows). On the lateral tumor border, arterial enhancement is not clearly seen on the grayscale images (B, $C$; white arrows) but is easily detectable on the arterial enhancement (AEF) map (D; white arrow). On the corresponding histopathologic images, vital HCC cells (E, F; white arrows), hypervascularity (F; black asterisk), and normal hepatocytes can be seen (E; black arrow). A, unenhanced scan; B, arterial phase; C, portal venous phase; $D$, arterial enhancement fraction (AEF) map; $E$ and F, corresponding hematoxylin and eosin-stained histopathologic images. 

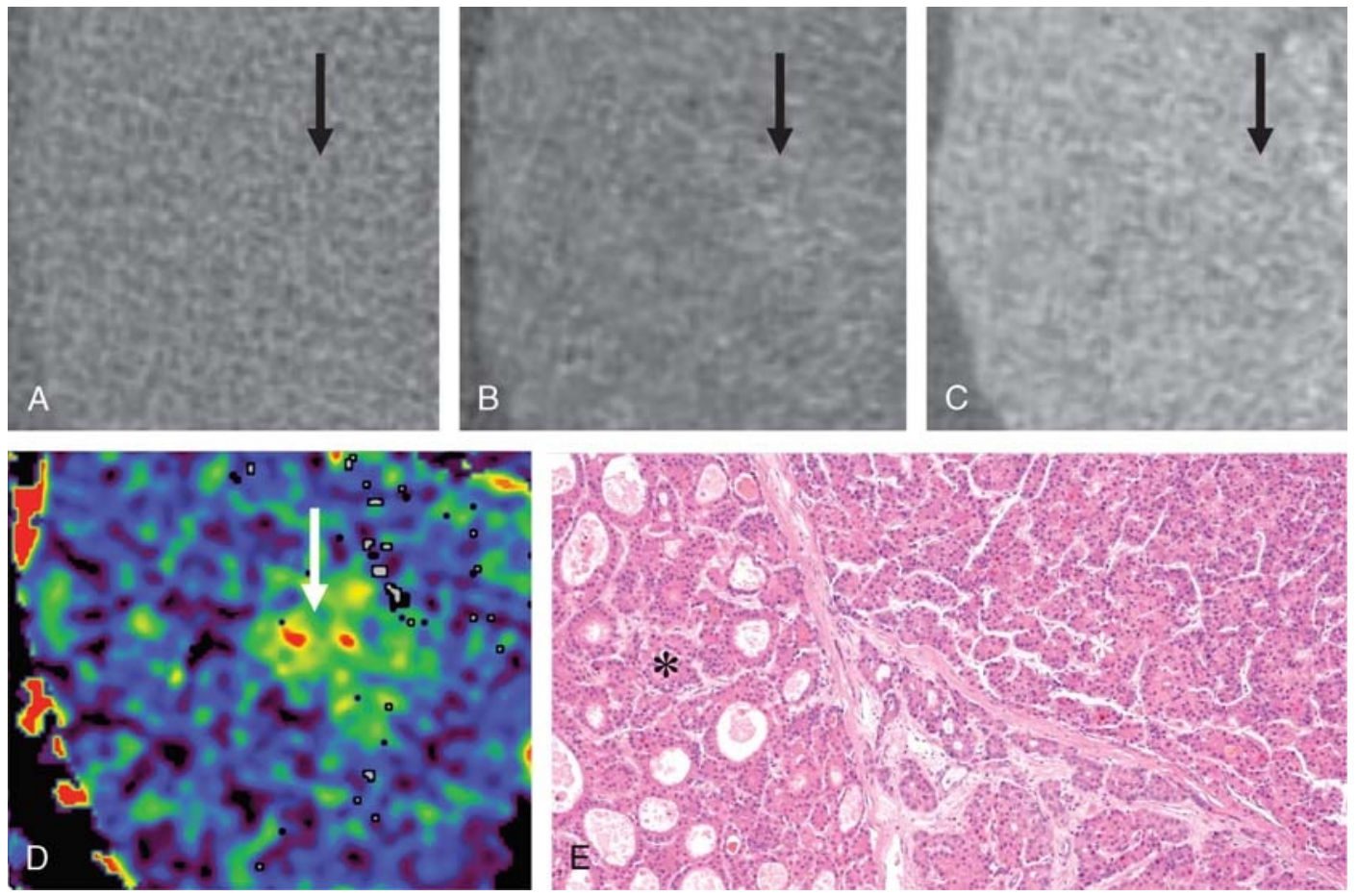

FIGURE 3. Well-differentiated HCC that is difficult to detect on the grayscale images with slight enhancement in the arterial phase and a minimal washout in the portal venous phase (A, B, C; black arrows). The arterial enhancement (AEF) map allows detection of the hypervascular lesion (D; white arrow). On the corresponding hematoxylin and eosin-stained histopathologic images, well-differentiated HCC cells (E) with acinar (black asterisk) and trabecular growth pattern (white asterisk) are seen. A, unenhanced scan; B, arterial phase; C, portal venous phase; D, arterial enhancement fraction (AEF) map; E, corresponding hematoxylin and eosin-stained histopathologic images.

and a specificity of $92.8 \%(95 \%$ CI, $91.6 \%-94.0 \%)$. The area under the curve was 0.957 . Figures 2 and 3 show examples of HCC lesions with the corresponding AEF map and histopathologic images.

The JAFROC analysis showed a significant difference in the figures of merit $(\theta)$ between the triphasic conventional readout $(\theta=0.6935)$ and the triphasic readout with additional availability of the AEF $(\theta=0.7478)$ in group 1 , with a $P$ value of 0.0002 . For group 2 , there was a slightly, but not significantly, higher figure of merit of the triphasic readout with additional availability of the $\operatorname{AEF}(\theta=0.7615)$ compared with the fourphasic conventional readout $(\theta=0.7569)$, with a $P$ value of 0.7541. A comparison of the AEF readout between the 2 groups of readers, which consisted of identical analysis of triphasic conventional images with additional AEF maps, was minimal $(P=0.3482)$, showing similar readout performance of the 2 groups of readers. The conventional triphasic readout of group $1(\theta=0.6935)$ was significantly inferior to the conventional fourphasic conventional readout of group

TABLE 1. Results of the JAFROC Analysis

\begin{tabular}{lccr}
\hline & Without AEF & With AEF & $\boldsymbol{P}$-value \\
\hline Group 1 & $\theta=0.69$ & $\theta=0.75$ & $<0.01$ \\
Group 2 & $\theta=0.76$ & $\theta=0.76$ & 0.75 \\
$P$ & $<0.01$ & 0.35 & \\
\hline
\end{tabular}

Group 1 looked at 3 contrast acquisitions (unenhanced, arterial, and portal venous) during the conventional readout, whereas group 2 had 4 contrast acquisitions (unenhanced, arterial, portal venous, and equilibrium phase) available. $\theta=$ figure of merit.

AEF indicates arterial enhancement fraction. $2(\theta=0.7569)$, with a $P$ value of $<0.0001$. The results of the JAFROC analysis are shown in Table 1.

In the group looking at triphasic $\mathrm{CT}$ scans, $\mathrm{HCC}$ was detected with a sensitivity of $30.7 \%(95 \%$ CI, $25.5 \%-36.4 \%)$ and a PPV of $72.0 \%(95 \%$ CI, 63.3\%-79.7\%) without the AEF, compared with a sensitivity of $47.7 \%(95 \% \mathrm{CI}, 41.9 \%-53.5 \%)$ and a PPV of $82.2 \%$ (95\% CI, 75.7\%-87.6\%) including the AEF. In the group looking at fourphasic $\mathrm{CT}$ acquisitions, HCC lesions were detected with a sensitivity of $42.1 \%(95 \% \mathrm{CI}, 36.2 \%-48.1 \%)$ and a PPV of $80.1 \%(95 \% \mathrm{CI}$, $72.7 \%-86.3 \%$ ) without the AEF, compared with a sensitivity of $49.8 \%$ (95\% CI, 43.9\%-55.8\%) and a PPV of $84.0 \%$ (95\% CI, 77.6\%-89.2\%) with the AEF (Table 2).

\section{Interreader Variability}

The $\kappa$ statistic showed a moderate mean unweighted interreader variability value of 0.42 without the AEF map $(\kappa=0.37 /$ fair for the triphasic readout and $\kappa=0.48 /$ moderate for the fourphasic readout), compared with a substantial mean unweighted interreader variability value of $\kappa=0.62$ for the readout with the AEF map including all 6 readers.

\section{Correlation of the Measured AEF Values With Serum Alpha-Fetoprotein Levels}

There was a very good correlation of the sum of the lesion diameters with AEF values greater than $50 \%$ with the sum of the lesion diameters measured by the hepatopathologist $(r=0.85, P<0.01)$ (Table 3). On the other hand, there was a rather poor correlation between the measured serum alpha-fetoprotein levels and the AEF values ( $r=0.37, P=0.01)$, as well as with the total HCC diameter measured by the hepatopathogist $(r=0.33, P=0.03)$. 
TABLE 2. Comparison of the Sensitivity, the Specificity and the Positive Predictive Value (Precision) of the Different Readouts

\begin{tabular}{|c|c|c|c|c|}
\hline & \multicolumn{2}{|c|}{ Group 1} & \multicolumn{2}{|c|}{ Group 2} \\
\hline & Triphasic CT Acquisition & Triphasic CT Acquisition & Triphasic CT Acquisition & Fourphasic CT Acquisition \\
\hline & With AEF & Without AEF & With AEF & Without AEF \\
\hline Sensitivity & $47.7 \%(41.9 \%-53.5 \%)$ & $30.7 \%(25.5 \%-36.4 \%)$ & $49.8 \%(43.9 \%-55.8 \%)$ & $42.1 \%(36.2 \%-48.1 \%)$ \\
\hline Specificity & $97.4 \%(96.4 \%-98.3 \%)$ & $97.1 \%(96.0 \%-98.0 \%)$ & $97.6 \%(96.6 \%-98.4 \%)$ & $97.5 \%(96.4 \%-98.3 \%)$ \\
\hline Positive predictive value & $82.2 \%(75.7 \%-87.6 \%)$ & $72.0 \%(63.3 \%-79.7 \%)$ & $84.0 \%(77.6 \%-89.2 \%)$ & $80.1 \%(72.7 \%-86.3 \%)$ \\
\hline Interreader variability $\kappa$ & 0.59 & 0.37 & 0.63 & 0.48 \\
\hline
\end{tabular}

The mean interreader variability $\kappa$ between the readers in each group is shown on the last line for the 2 readouts $(\kappa$ value $0-0.2$, poor; $0.21-0.4$, fair; $0.41-0.6$, moderate; $0.61-0.8$, substantial; $0.81-1$, almost perfect). For the first readout, every reader in both groups looked at the same AEF maps in adjunct to the triphasic CT acquisitions (unenhanced, arterial, and portal venous phase). For the second readout, the AEF maps were not shown to the readers. The readers in group 1 looked at triphasic CT acquisitions, whereas group 2 had 4 contrast acquisitions available (unenhanced, arterial, portal venous, and equilibrium phase). The $95 \%$ confidence interval is indicated in parenthesis.

AEF indicates arterial enhancement fraction; TACE, transarterial chemoembolization.

TABLE 3. Correlation Between the Measured Serum Alpha-Fetoprotein Levels, AEF Values, and the Sum of the Lesion Diameters as Measured by the Hepatopathologist

\begin{tabular}{lcc}
\hline & $\begin{array}{c}\text { Sum of the Diameters of the Lesions } \\
\text { With an AEF } \mathbf{5 0 \%}\end{array}$ & $\begin{array}{c}\text { Sum of the Lesion Diameters as } \\
\text { Measured by the Hepatopathologist }\end{array}$ \\
\hline Serum alpha-fetoprotein levels & $r=0.37(P=0.01)$ & $r=0.33(P=0.03)$ \\
Sum of the lesion diameters as & $r=0.85(P<0.01)$ & \\
measured by the hepatopathologist & & \\
\hline
\end{tabular}

AEF indicates arterial enhancement fraction.

\section{Detection of HCC in Segments With and Without Previous Tace}

In liver segments with previous TACE treatment, HCC lesions were detected with a sensitivity of $31.9 \%(95 \%$ CI, $26.8 \%-37.3 \%)$ and a PPV of $85.1 \%$ (95\% CI, 77.5\%-90.9\%) without the use of the AEF map and with a sensitivity of $48.4 \%$ (95\% CI, 42.9\%-53.9\%) and a PPV of $91.5 \%(95 \%$ CI, 86.4\%-95.2\%) with the AEF perfusion maps. Lesions in liver segments without previous TACE treatment, on the other hand, have been detected with a sensitivity of $42.3 \%(95 \%$ CI, 36.1\%-48.8\%) and a PPV of 69.5\% (95\% CI, 61.5\%-76.8\%) without the use of the AEF map and with a sensitivity of $51.6 \%$ (95\% CI, 45.2\%$57.9 \%)$ and a PPV of $75.0 \%$ (95\% CI, 67.8\%-81.3\%) with the AEF map (Table 4). Figure 4 shows an example of a recurrent HCC after previous TACE with the corresponding AEF map and histopathologic images.

\section{DISCUSSION}

The use of the AEF significantly improved the sensitivity and the precision (PPV) of radiologists in HCC detection in a setting of patients with advanced liver cirrhosis on the liver transplantation list.

On the basis of the presented data, we suggest a cutoff AEF value of $50 \%$, which is comparable with the results of Lee et al, ${ }^{21}$ who calculated a cutoff value of $45 \%$ in their analysis. We assume our result to be more precise, since Lee et al used a per-lesion-based analysis, allowing to count either true-positive or false-negative lesions, ignoring AEF measurements in true-negative liver segments. This might be the reason why our cutoff value is slightly higher.

With the use of the AEF map, the performance of the radiologists in detecting HCC lesions could be significantly improved, and interreader variability was increased from moderate without the AEF maps to substantial with addition of the AEF maps. In fact, the additional use of AEF maps could compensate the additive value of a fourth CT contrast acquisition in equilibrium phase. This might allow superseding the fourth contrast acquisition in the equilibrium phase, which would allow a dose reduction of $25 \%$. This could be of interest to reduce cumulative radiation dose for patients with chronic liver disease undergoing repetitive HCC screening with CT. There was a benefit of

TABLE 4. Comparison of the Mean Sensitivity, Specificity, and Positive Predictive Value (Precision) of all Readers Between Liver Segments With Previous TACE and Liver Segments Without Previous TACE

\begin{tabular}{|c|c|c|c|c|}
\hline & \multicolumn{2}{|c|}{ With Previous TACE } & \multicolumn{2}{|c|}{ No Previous TACE } \\
\hline & Without AEF & With AEF & Without AEF & with AEF \\
\hline Sensitivity & $31.9 \%(26.8 \%-37.3 \%)$ & $48.4 \%(42.9 \%-53.9 \%)$ & $42.3 \%(36.1 \%-48.8 \%)$ & $51.6 \%(45.2 \%-57.9 \%)$ \\
\hline Specificity & $96.2 \%(94.1 \%-97.7 \%)$ & $96.8 \%(94.7 \%-98.2 \%)$ & $97.6 \%(96.8 \%-98.2 \%)$ & $97.7 \%(96.9 \%-98.4 \%)$ \\
\hline Positive predictive value & $85.1 \%(77.5 \%-90.9 \%)$ & $91.5 \%(86.4 \%-95.2 \%)$ & $69.5 \%(61.5 \%-76.8 \%)$ & $75.0 \%(67.8 \%-81.3 \%)$ \\
\hline
\end{tabular}

The $95 \%$ confidence interval is indicated in parenthesis.

AEF indicates arterial enhancement fraction; TACE, transarterial chemoembolization. 

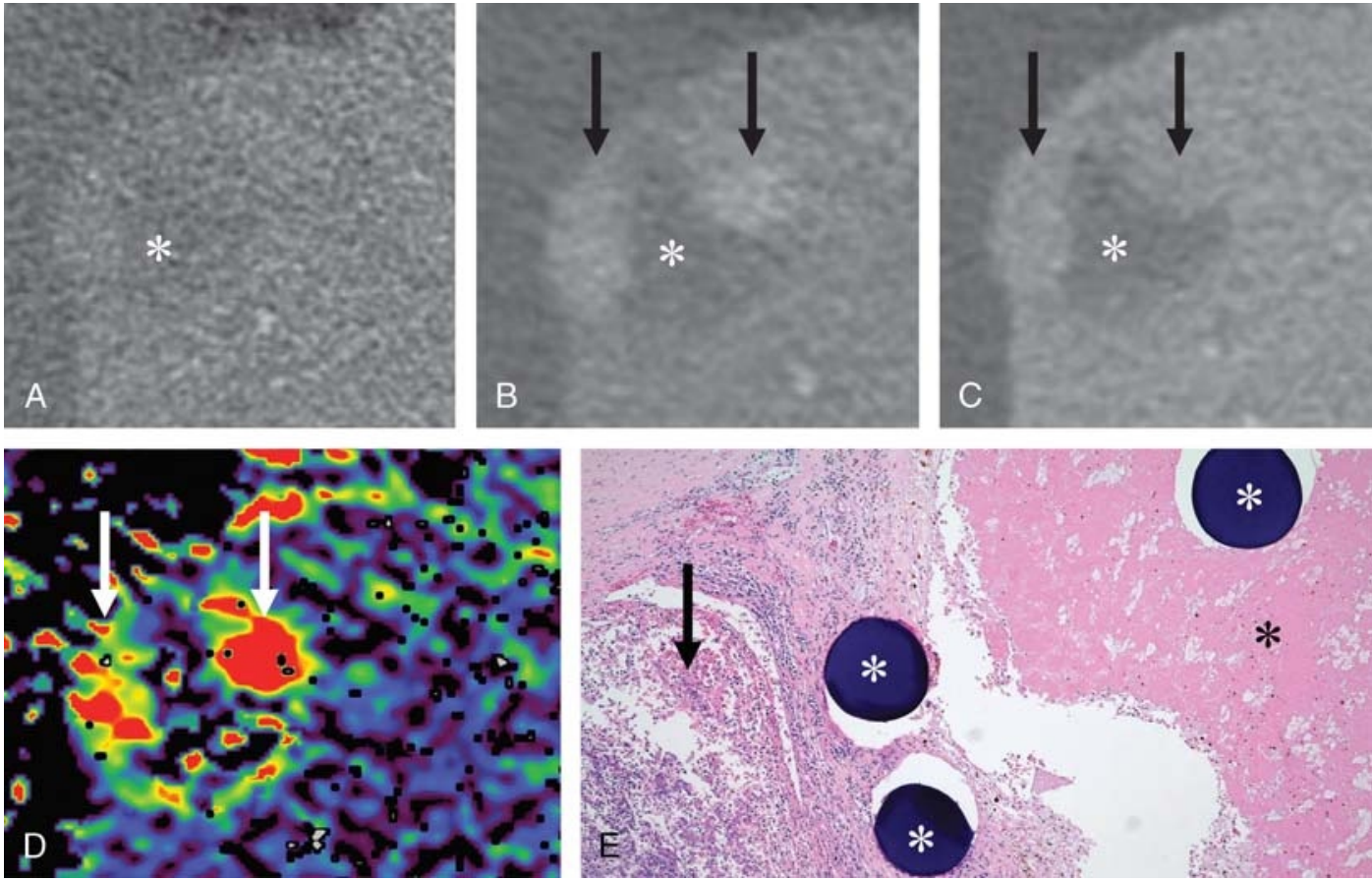

FIGURE 4. Recurrent HCC after TACE. After TACE, there is a tumor necrosis in the center of the lesion (A, B, C; white asterisk), which can also be seen in the corresponding histopathologic images (E; black asterisk). Recurrent nodular HCC on the medial and lateral border of the necrosis shows arterial enhancement, but no clear washout in the portal venous phase. The lateral tumor border infiltrates the liver capsule (B, C; black arrows). The arterial enhancement (AEF) map is positive, consistent with HCC activity (D; white arrows). On the corresponding hematoxylin and eosin-stained histopathologic images, vital HCC cells (E; black arrow) and the microspheres after TACE (E; white asterisk) are seen. A, unenhanced scan; B, arterial phase; $C$, portal venous phase; D, arterial enhancement fraction (AEF) map; $E$, corresponding hematoxylin and eosin-stained histopathologic image.

using the AEF no matter if the lesion was located in liver segments with or without previous TACE. However, there was an overall difference in the detection of HCC lesions located in liver segments with previous TACE compared with lesions located in segments without previous TACE; lesions in segments with previous TACE were detected with a lower sensitivity $(31.89 \%$ without the AEF vs $48.36 \%$ with the AEF) than HCC lesions located elsewhere (42.34\% without the AEF vs $51.60 \%$ with the AEF). This can be explained by the reduced arterial blood supply even if viable residual HCC tissue is present in this area, once the arterial feeders have been embolized. ${ }^{22}$ The portal venous blood supply, on the other hand, rests unchanged. This results in a reduction in arterial enhancement as well as a reduction in the washout in the portal and the equilibrium phase. Inversely, HCC lesions located in liver segments with previous TACE have been detected with a higher PPV $(85.12 \%$ without the AEF vs $91.53 \%$ with the AEF) than HCC lesions located elsewhere $(69.54 \%$ without the AEF vs $75.00 \%$ with the AEF). This seems to be logical because a nodule in an area where there was already an HCC is more likely to be an HCC than in an area where there was no HCC.

In a setting of chronic liver disease, any lesion with arterial enhancement and washout on the grayscale images should be diagnosed as HCC. Because these imaging characteristics are not always very well identifiable, the AEF can help to raise confidence for HCC in cases of weak arterial enhancement and/or uncertain washout. It is important to note that the AEF is not a new imaging characteristic for $\mathrm{HCC}$ but a tool that may help to better identify the known perfusion characteristics of HCC. The AEF thus allows not only to detect lesions that have been overlooked in the conventional readout but also to raise the sensitivity by including HCC lesions with weak arterial enhancement and/or uncertain washout without losing the high specificity of $96 \%$ to $98 \%$. This effect is reflected in a higher PPV when using the AEF maps. Or in other words, if lesions with weak arterial enhancement and uncertain washout are called positive in an HCC screening setting to increase the detection rate, the specificity will drop. The additional use of the AEF helps to re-raise the specificity to the initial level. Two other major signs to affirm the diagnosis of HCC beneath the perfusion characteristics are the presence of a capsule and threshold growth between 2 imaging studies. The data published by Yu et $\mathrm{al}^{5}$ investigating CT detection rate in liver transplant recipients showed a higher sensitivity of $65 \%$ for HCC-detection in fourphasic CT scans compared with this present study, with a sensitivity of $42 \%$ for fourphasic acquisition without AEF. There are different reasons that might explain this difference. One reason is that $\mathrm{Yu}$ et al rated any liver lesion, even if it was not clearly characterized as benign, as HCC. In the present study, only lesions with a confidence score of 3 of 5 given by the readers were called positive. Another possible explanation for this difference is that $\mathrm{Yu}$ et al excluded all HCC lesions with previous TACE. Last but not least, the radiologists in the study of Yu et al were not blinded to other imaging results, including ultrasound, CT, and MRI studies, and did have knowledge of clinical information such as the serum alpha-fetoprotein levels. The present study was designed to investigate the additional value of the AEF, and the readers have thus been completely blinded to any clinical information and were not allowed to look at previous or complementary imaging studies. However, in a realistic setting of HCC screening, the radiologists will always correlate the results to previous/ complementary imaging studies as well as clinical information and thus might achieve better $\mathrm{HCC}$ detection rates than presented in this article. Another study by Lee et $\mathrm{al}^{23}$ analyzing the additional use of AEF perfusion maps to detect HCC lesions with previous TACE also showed a higher detection rate for the HCC lesions on fourphasic $\mathrm{CT}$ acquisition (sensitivity of $62.7 \%$, compared with $42.1 \%$ in this study), as well as on the fourphasic CT with additional use of AEF maps (sensitivity 
of $82.1 \%$, compared with $49.8 \%$ in this study). The most striking difference between the 2 studies' methods is that Lee et al did not have a gross pathologic explant as the gold standard to determine the true-positive lesions because they did not investigate patients before liver transplantation. Instead, they used the recommendations from the AASLD ${ }^{14}$ to define positive HCC lesions. Many lesions that have been undetected clinically and on CT scans thus may not have been included, which might explain the difference in performance compared with the present study. The results of this study are confirmed by the paper of Peterson et $\mathrm{al}^{24}$ who investigated triphasic $\mathrm{CT}$ detection rates before liver transplantation compared with the histological analysis of the liver explants. Their results showed a sensitivity of $29 \%$ for the HCC lesions in the prospective clinical reports, which is comparable with the sensitivity of $30.7 \%$ for the triphasic $\mathrm{CT}$ acquisitions without the AEF maps in the present study.

With or without the AEF maps, the sensitivity for HCC detection remained low in a population of patients with end-stage liver disease on the liver transplantation list. Because HCC is often multifocal, the per-patient sensitivity in a screening population will be higher than the per-lesion sensitivity calculated in this study. Furthermore, small HCC less than $2 \mathrm{~cm}$ will grow over time and might be detected in the follow-up imaging study. Also, the radiologists should know the serum alpha-fetoprotein level while reading the CT scans to achieve a better screening performance, which was not the case in this study. In case of an increased serum alpha-fetoprotein level and a negative CT scan, perfusion MRI with gadoxetic acid and diffusion weighted imaging allows to raise sensitivity for HCC detection up to $78.8 \%$ in a comparable patient population with end-stage liver disease for an experienced radiologist in the study of Hwang et al. ${ }^{25}$ However, bad clinical conditions of patients on the liver transplantation list and presence of ascites in these patients are major limitations for MRI studies.

The rather poor correlation between the serum alpha-fetoprotein levels and the measured AEF values, as well as with the sum of the HCC lesion diameter as measured by the hepatopathologist, can be explained mainly by the heterogeneous tumor activity of $\mathrm{HCC}$ and the fact that the alpha-fetoprotein level cannot be measured separately for each HCC lesion. It is rather a marker for the total activity of the HCC mass in 1 patient. Also, there are some limits in sensitivity and specificity of the alpha-fetoprotein for HCC. ${ }^{26}$ However, there was a very good correlation between the total HCC diameter in pathology and the total lesion diameter with an AEF greater than 50\%. A further study comparing the sum of AEF values per tumor volume with the serum tumor markers in a larger series of patients would be very promising.

Hepatocellular carcinoma screening is recommended by most societies for chronic liver disease, whereas normally a screening interval of 6 months with ultrasound and biomarkers is advised. ${ }^{27}$ However, knowing the low detection rates for small HCC lesions with ultrasound in a patient population with end-stage liver disease, CT or MRI is recommended by the AASLD in patients with a hepatic nodule bigger than $1 \mathrm{~cm} .{ }^{14}$ Also, it might be used in obese patients who are difficult to examine with ultrasound, ${ }^{28}$ as well as in patients with previous, treated HCC. ${ }^{29}$ There is a new recommendation for liver imaging reporting and data system (Liver Imaging Reporting and Data System) by the American College of Radiology to standardize the reporting and data collection of CT and MRI for HCC in these patients. ${ }^{30}$ Beyond the arterial phase hyperenhancement and the washout phenomenon, it also takes into account a threshold growth of the lesion and the presence or absence of a capsule around the lesion. ${ }^{31}$

\section{Limitations}

A major weakness of this study is its retrospective nature and the homogenous patient population with end-stage liver cirrhosis on the OLT list. In a patient population with less advanced chronic liver disease, the detection rate for HCC would probably be higher because patients with end-stage liver disease will show a more heterogeneous liver enhancement with the presence of more regenerative nodules. Thus, the resulting calculated AEF maps are also likely to be more heterogeneous than the AEF maps of patients with less advanced chronic liver disease. Another factor is the reduced general condition with difficult image acquisition in the present patient population due to motion artifacts and ascites. Although the parenchyma of the cirrhotic livers is very stiff, there will always be some deformation of the liver parenchyma in the presence of respiration between the different CT acquisitions. The different acquisitions will never match perfectly to calculate AEF maps, even in case of an optimal motion correction.

Another weakness of the study is that the readers were grouped into 1 group looking at 3 contrast phases (unenhanced, arterial, and portal venous phase) and 1 group looking at 4 contrast phases (unenhanced, arterial, portal venous, and equilibrium phase) during the conventional readout. This was done to allow a comparison of 3 versus 4 contrast phases but diminishes the statistical power of the study. Nevertheless, the results have been significant; the detection rate of the triphasic CT acquisitions without the AEF of group 1 were compatible with the results of Peterson et al, who also analyzed detection rates of HCC lesions in triphasic CT scans of liver transplant recipients with direct pathologic correlation to the liver explants. There was no significant difference in performance between the 2 reader groups comparing the 2 readouts using the AEF perfusion maps. The $\kappa$ statistic showed a substantial mean unweighted interreader variability between the 6 readers for the triphasic readout with the AEF tool.

The fact that the readers were completely blinded to complementary/ previous imaging studies and clinical information was intended for the objectives of this study, but radiologists will always consider clinical information and compare it with previous imaging studies in a real setting of HCC screening. This could lead to a better HCC detection rate than shown in this study. A possible use of the AEF maps in combination with 4 contrast acquisitions has not yet been analyzed. This could be of interest to additionally increase detection rates and should be done in a further, optimally prospective study.

\section{CONCLUSION}

This radiologic-pathologic correlation study shows that AEF is a helpful tool to screen for HCC in patients on the OLT list. We recommend a cutoff value of $50 \%$ for the AEF. The use of AEF maps may significantly improve sensitivity and precision (PPV) of HCC detection and a decrease interreader variability.

There was no significant difference in performance of a triphasic CT using AEF compared with a fourphasic CT without AEF. This might allow a $25 \%$ reduction in radiation dose, which is relevant for patients undergoing repetitive HCC screening with $\mathrm{CT}$.

Lesions located in liver segments with previous TACE have been detected with a lower sensitivity than lesions located in liver segments without previous TACE. On the contrary and not surprisingly, the PPV was better in liver segments with previous TACE. In both scenarios, a beneficial use of the AEF perfusion maps has been shown.

\section{REFERENCES}

1. Jemal A, Bray F, Center MM, et al. Global cancer statistics. CA Cancer J Clin. 2011;61:69-90.

2. Sherman M, Bruix J, Porayko M, et al. Screening for hepatocellular carcinoma: the rationale for the American Association for the Study of Liver Diseases recommendations. Hepatology. 2012;56:793-796.

3. Ganslmayer M, Hagel A, Dauth W, et al. A large cohort of patients with hepatocellular carcinoma in a single European centre: aetiology and prognosis now and in a historical cohort. Swiss Med Wkly. 2014;144:w13900. Available at: http://www.smw.ch/content/smw-2014-13900/. Accessed August 22, 2014.

4. El-Serag HB. Surveillance for hepatocellular carcinoma: long way to achieve effectiveness. Dig Dis Sci. 2012;57:3050-3051. 
5. Yu NC, Chaudhari V, Raman SS, et al. CT and MRI improve detection of hepatocellular carcinoma, compared with ultrasound alone, in patients with cirrhosis. Clin Gastroenterol Hepatol. 2011;9:161-167.

6. Crissien AM, Frenette C. Current management of hepatocellular carcinoma. Gastroenterol Hepatol. 2014;10:153-161.

7. Fischer MA, Leidner B, Kartalis N, et al. Time-resolved computed tomography of the liver: retrospective, multi-phase image reconstruction derived from volumetric perfusion imaging. Eur Radiol. 2014;24:151-161.

8. Pandharipande PV, Krinsky GA, Rusinek H, et al. Perfusion imaging of the liver: current challenges and future goals. Radiology. 2005;234:661-673.

9. Fournier LS, Cuenod CA, de Bazelaire C, et al. Early modifications of hepatic perfusion measured by functional $\mathrm{CT}$ in a rat model of hepatocellular carcinoma using a blood pool contrast agent. Eur Radiol. 2004;14:2125-2133.

10. Wu D, Tan M, Zhou M, et al. Liver computed tomographic perfusion in the assessment of microvascular invasion in patients with small hepatocellular carcinoma. Invest Radiol. 2015;50:188-194.

11. Kim SH, Kamaya A, Willmann JK. CT perfusion of the liver: principles and applications in oncology. Radiology. 2014;272:322-344.

12. Kim KW, Lee JM, Klotz E, et al. Quantitative CT color mapping of the arterial enhancement fraction of the liver to detect hepatocellular carcinoma. Radiology. 2009;250:425-434.

13. Baron RL. Understanding and optimizing use of contrast material for CT of the liver. Am J Roentgenol. 1994;163:323-331.

14. Bruix J, Sherman M; Practical Guidelines Committee, American Association of Liver Disease. Management of hepatocellular carcinoma. Hepatology. 2005;42: 1208-1236.

15. Merion RM, Schaubel DE, Dykstra DM, et al. The survival benefit of liver transplantation. Am J Transplant. 2005;5:307-313.

16. Mazzaferro V, Regalia E, Doci R, et al. Liver transplantation for the treatment of small hepatocellular carcinomas in patients with cirrhosis. $N$ Engl J Med. 1996; 334:693-700.

17. Schoonjans F, Zalata A, Depuydt CE, et al. MedCalc: a new computer program for medical statistics. Comput Methods Programs Biomed. 1995;48:257-262.

18. Chakraborty DP. Analysis of location specific observer performance data: validated extensions of the jackknife free-response (JAFROC) method. Acad Radiol. 2006;13:1187-1193.

19. Light RJ. Measures of response agreement for qualitative data: some generalizations and alternatives. Psychol Bull. 1971;76:365-377.
20. Landis JR, Koch GG. An application of hierarchical kappa-type statistics in the assessment of majority agreement among multiple observers. Biometrics. 1977; 33:363-374.

21. Lee DH, Lee JM, Klotz E, et al. Detection of recurrent hepatocellular carcinoma in cirrhotic liver after transcatheter arterial chemoembolization: value of quantitative color mapping of the arterial enhancement fraction of the liver. Korean J Radiol. 2013;14:51-60.

22. Kan Z, Kobayashi S, Phongkitkarun S, et al. Functional CT quantification of tumor perfusion after transhepatic arterial embolization in a rat model. Radiology 2005;237:144-150

23. Lee DH, Kim SH, Lee JM, et al. Diagnostic performance of multidetector row computed tomography, superparamagnetic iron oxide-enhanced magnetic resonance imaging, and dual-contrast magnetic resonance imaging in predicting the appropriateness of a transplant recipient based on Milan criteria: correlation with histopathological findings. Invest Radiol. 2009;44:311-321.

24. Peterson MS, Baron RL, Marsh JW Jr, et al. Pretransplantation surveillance for possible hepatocellular carcinoma in patients with cirrhosis: epidemiology and CT-based tumor detection rate in 430 cases with surgical pathologic correlation. Radiology. 2000;217:743-749.

25. Hwang J, Kim YK, Kim JM, et al. Pretransplant diagnosis of hepatocellular carcinoma by gadoxetic acid-enhanced and diffusion-weighted magnetic resonance imaging. Liver Transpl. 2014;20(12):1436-1446.

26. Zhou Y, Yin X, Ying J, et al. Golgi protein 73 versus alpha-fetoprotein as a biomarker for hepatocellular carcinoma: a diagnostic meta-analysis. BMC Cancer. 2012;12:17.

27. Sherman M, Colombo M. Hepatocellular carcinoma screening and diagnosis. Semin Liver Dis. 2014;34:389-397.

28. El-Serag HB, Davila JA. Surveillance for hepatocellular carcinoma: in whom and how? Therap Adv Gastroenterol. 2011;4:5-10.

29. Liu D, Fong DY, Chan AC, et al. Hepatocellular carcinoma: surveillance CT schedule after hepatectomy based on risk stratification. Radiology. 2014;274: 133-140.

30. Mitchell DG, Bruix J, Sherman M, et al. LI-RADS (Liver Imaging Reporting and Data System): summary, discussion, and consensus of the LI-RADS Management Working Group and future directions. Hepatology. 2015;61:1056-1065.

31. Anon. American College of Radiology. Liver imaging reporting and data system version 2014. Available at: http://www.acr.org/Quality-Safety/Resources/LIRADS. Accessed May 2015. 